\title{
Effects of two fretting damage modes on dental implant-abutment interface and the generation of metal wear debris: an in vitro study
}

\author{
Jing $\mathrm{Gao}^{1}$, Jie $\mathrm{Min}^{1}$, Xin $\mathrm{Chen}^{2}$, Ping $\mathrm{Yu}^{2}$, Xin $\mathrm{Tan}^{2}$, Qianqian Zhang ${ }^{2}$, and Haiyang $\mathrm{Yu}^{1}$ \\ ${ }^{1}$ Sichuan University West China Hospital of Stomatology \\ ${ }^{2}$ Sichuan University West China College of Stomatology
}

June 12,2020

\begin{abstract}
The fretting damage and wear debris on the dental implant-abutment interface (IAI) are unclear. In this study, fatigue cycle loading (FT) and chewing cycle loading (CW) test were applied to two implant systems, the fretting damage morphology and wear debris generation on the IAI were observed by a scanning electron microscope. The torque value of the central screw was measured by electronic torque tester. The fretting damage on the IAI was relatively slight and mainly plastic deformation in the FT group, which was more serious and mainly furrow wear in the CW group. Various forms of wear debris were generated. The removal torques were lower than its pre-tightening value in both groups, the decline and loss rate of the CW group was significantly higher. This study confirmed the critical roles of fretting damages and metal wear debris on the IAI in the implant-supported prosthesis.
\end{abstract}

\section{Hosted file}

2020-06-09 title page.doc available at https://authorea.com/users/332889/articles/459238effects-of-two-fretting-damage-modes-on-dental-implant-abutment-interface-and-thegeneration-of-metal-wear-debris-an-in-vitro-study

\section{Hosted file}

2020-06-09 Highlights.docx available at https://authorea.com/users/332889/articles/459238effects-of-two-fretting-damage-modes-on-dental-implant-abutment-interface-and-thegeneration-of-metal-wear-debris-an-in-vitro-study

\section{Hosted file}

2020-06-15 Effects of two fretting damage modes on dental implant.docx available at https://authorea.com/users/332889/articles/459238-effects-of-two-fretting-damage-modeson-dental-implant-abutment-interface-and-the-generation-of-metal-wear-debris-an-invitro-study 

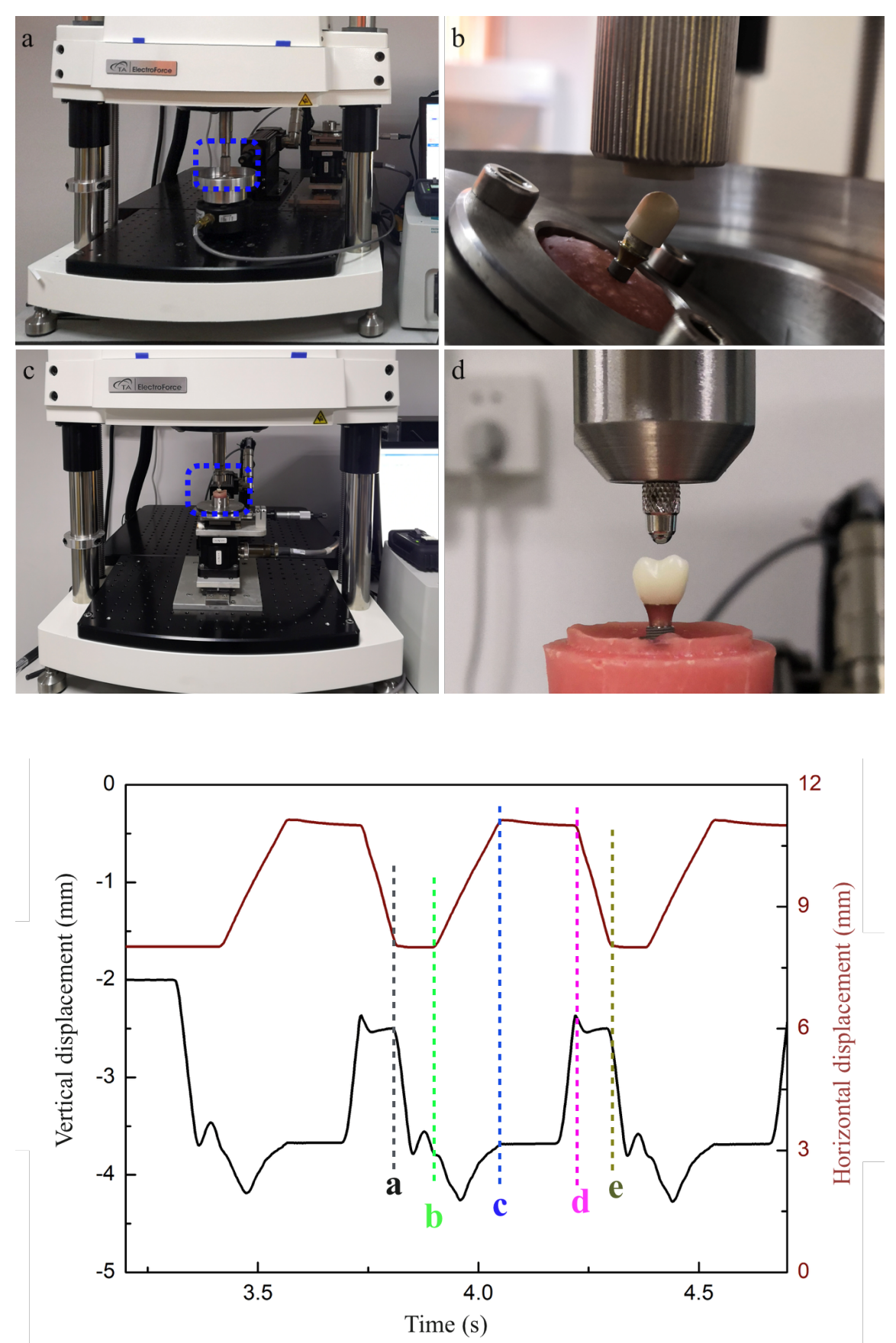


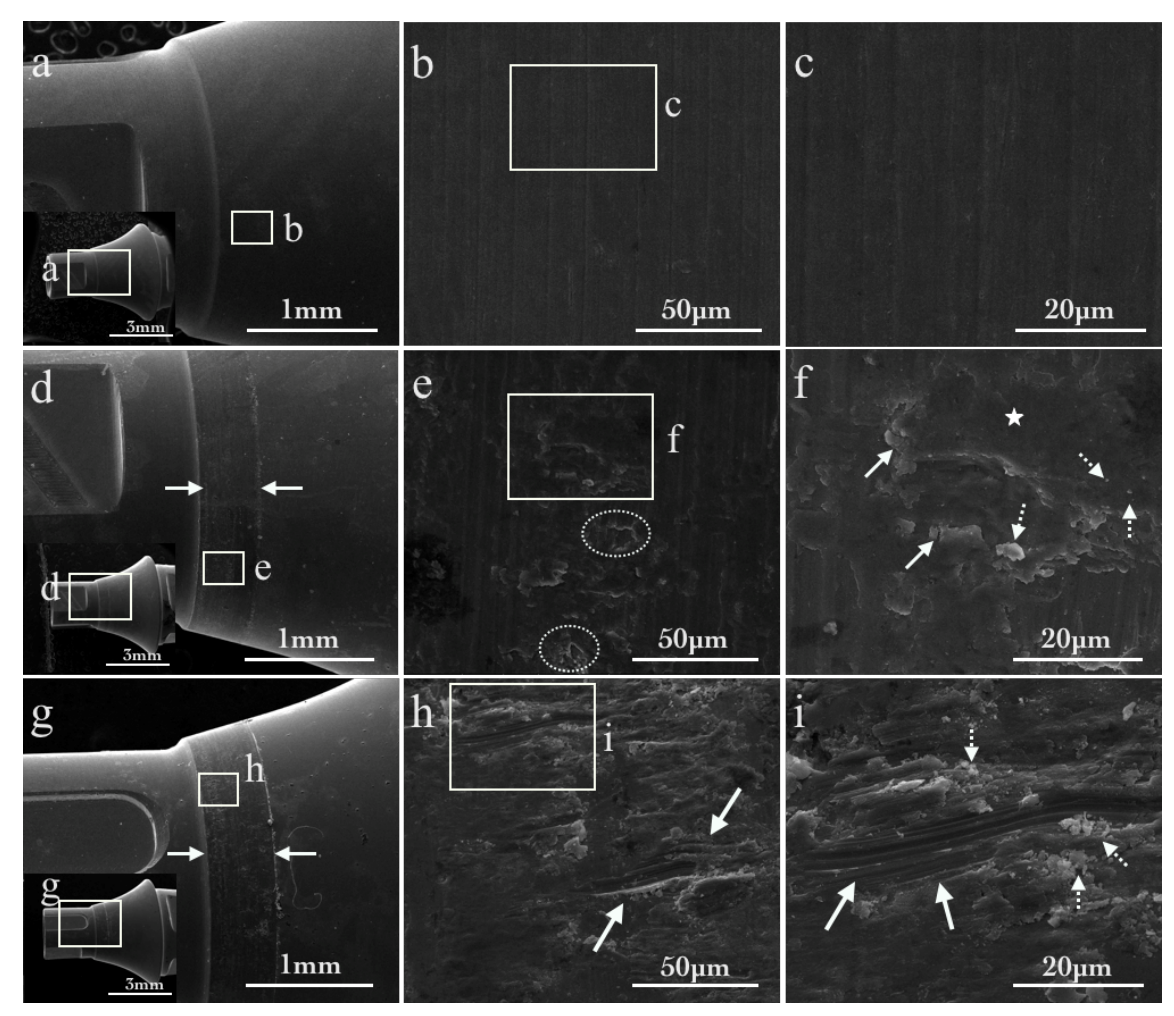




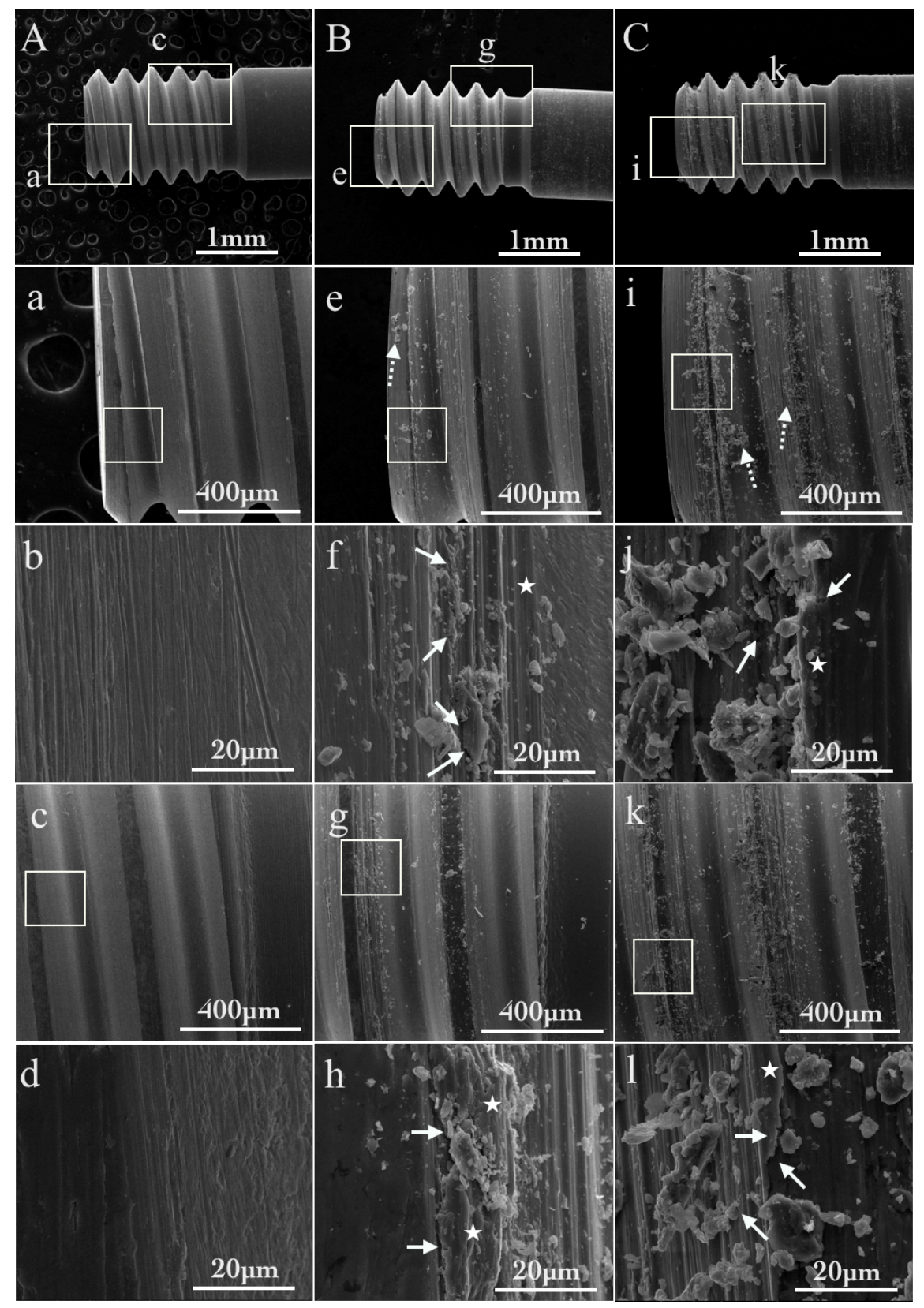




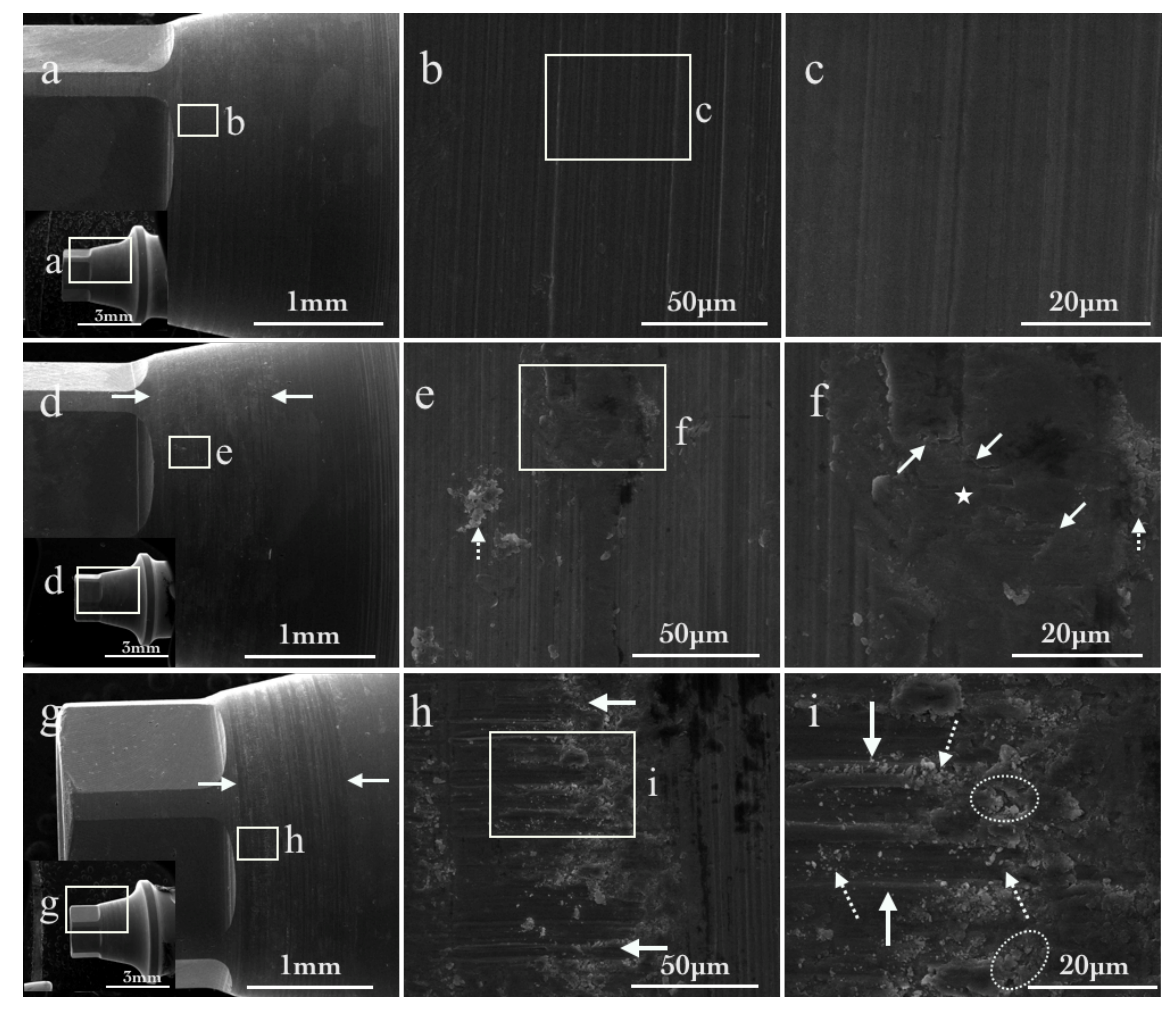




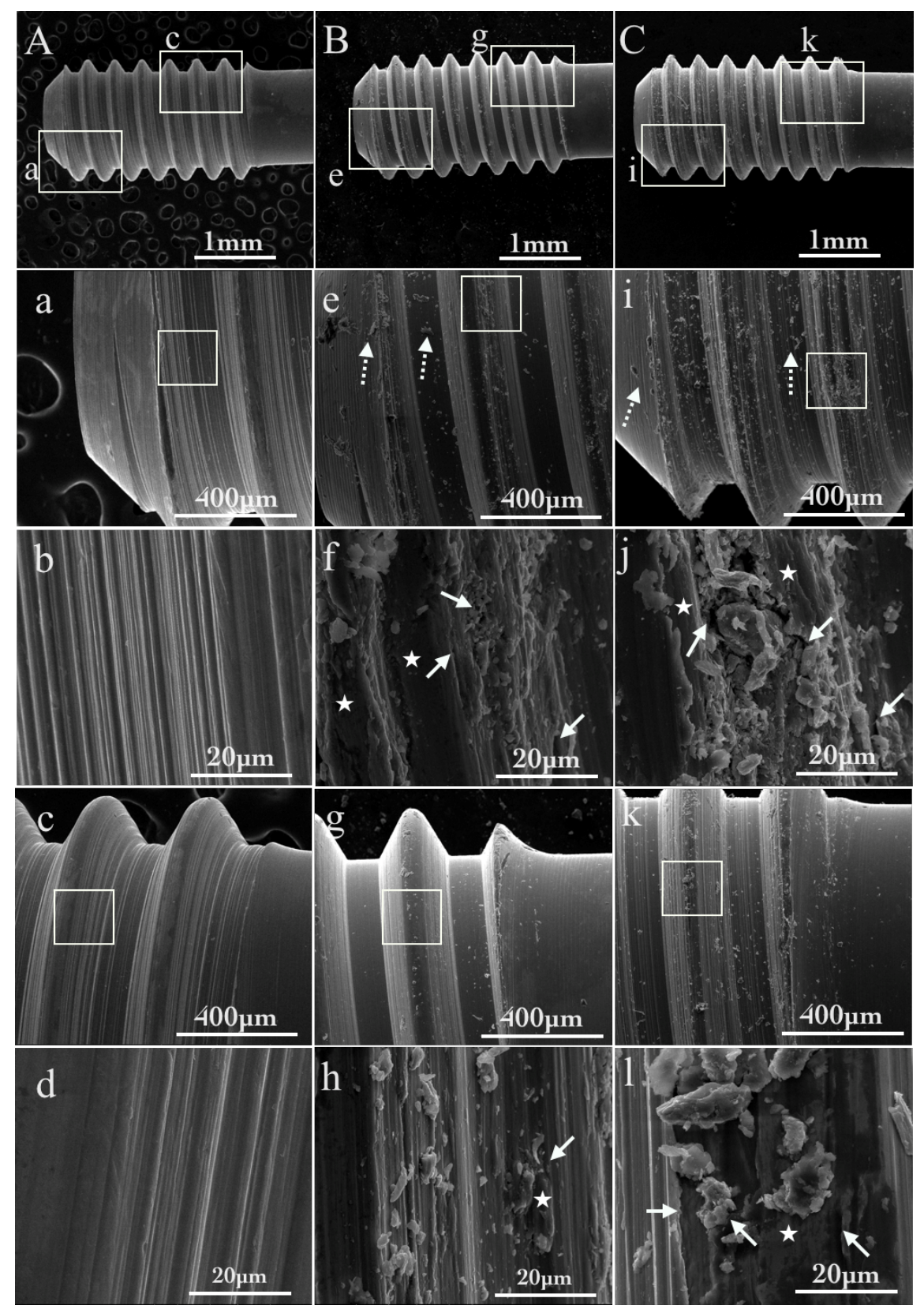




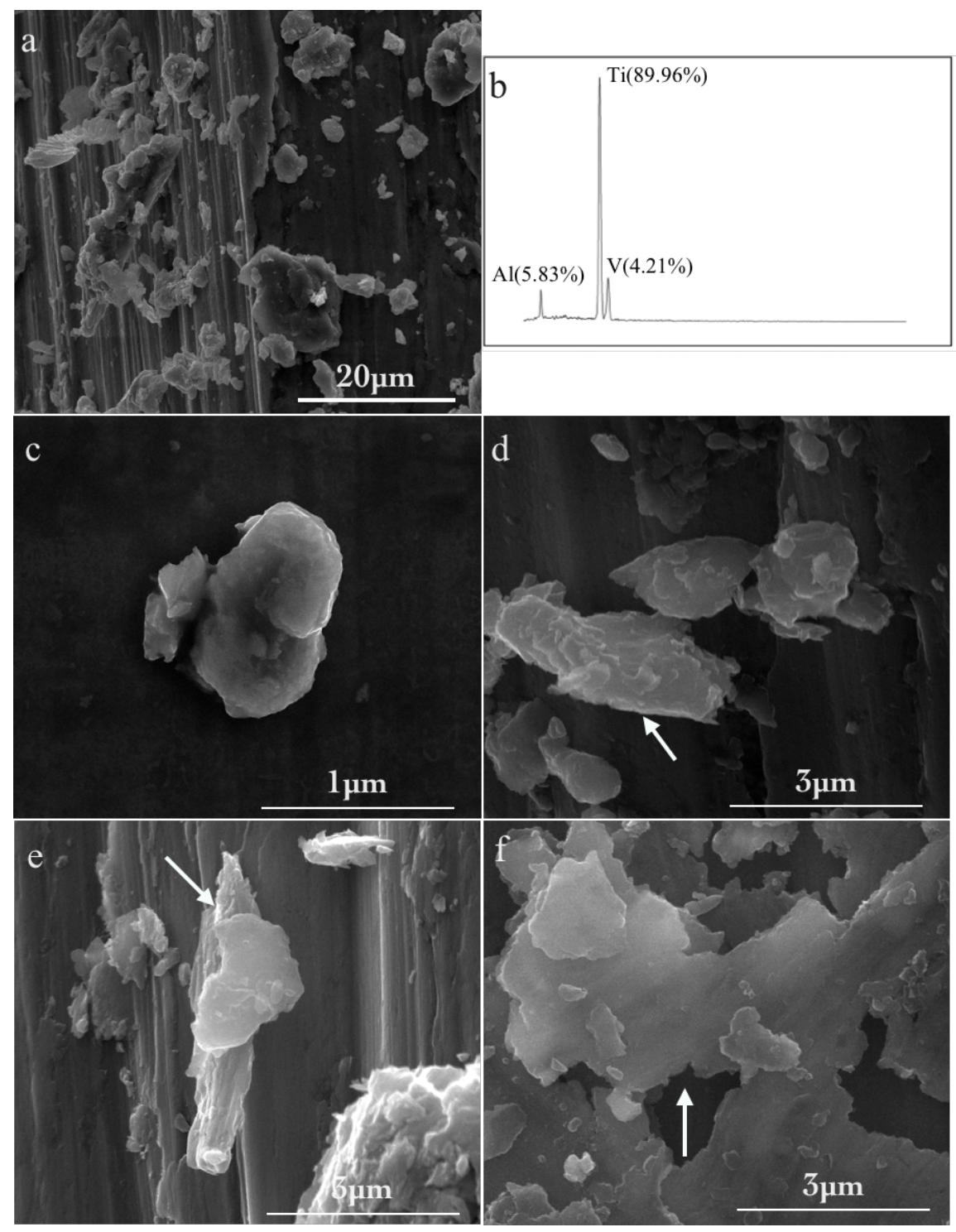



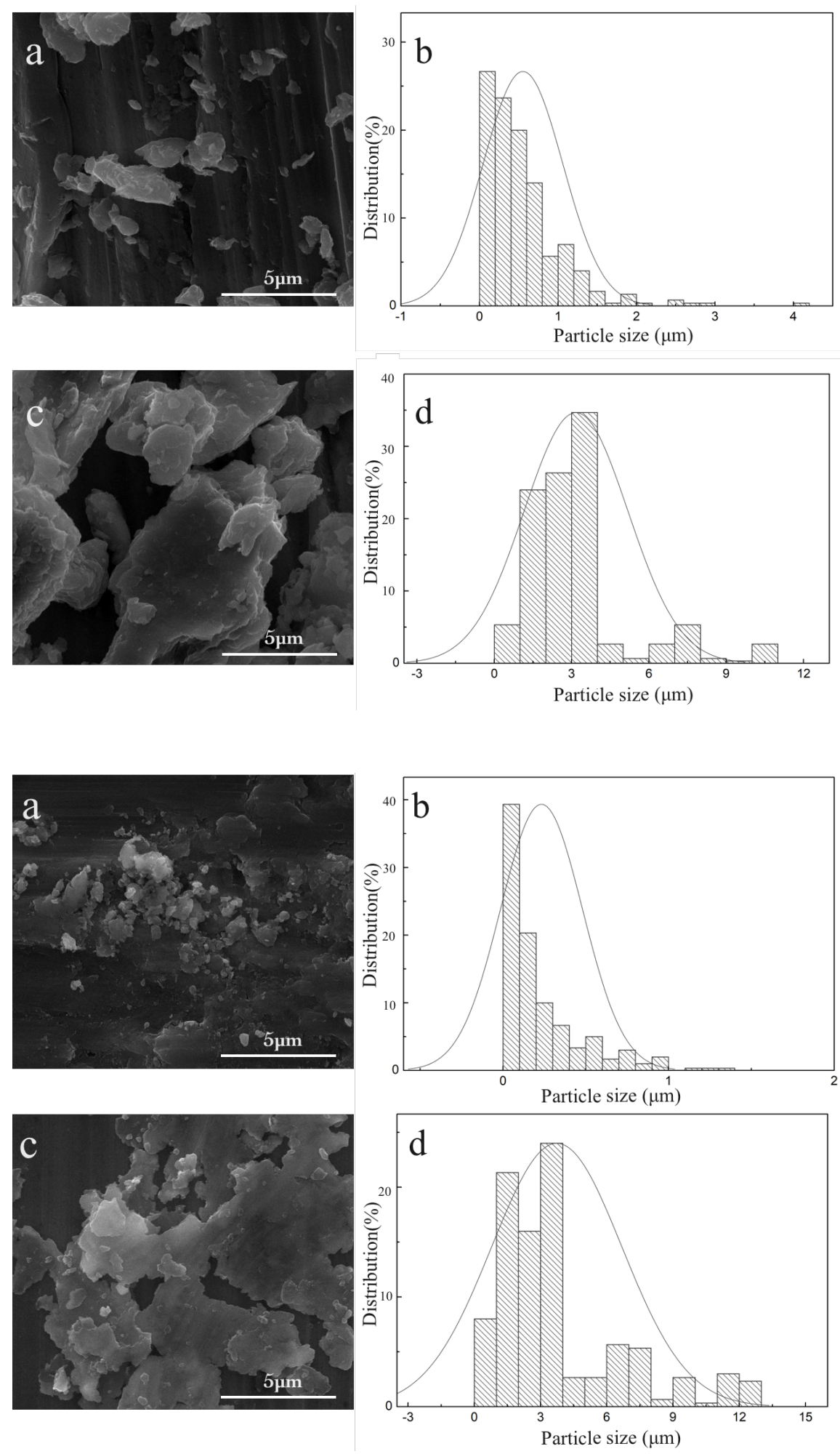

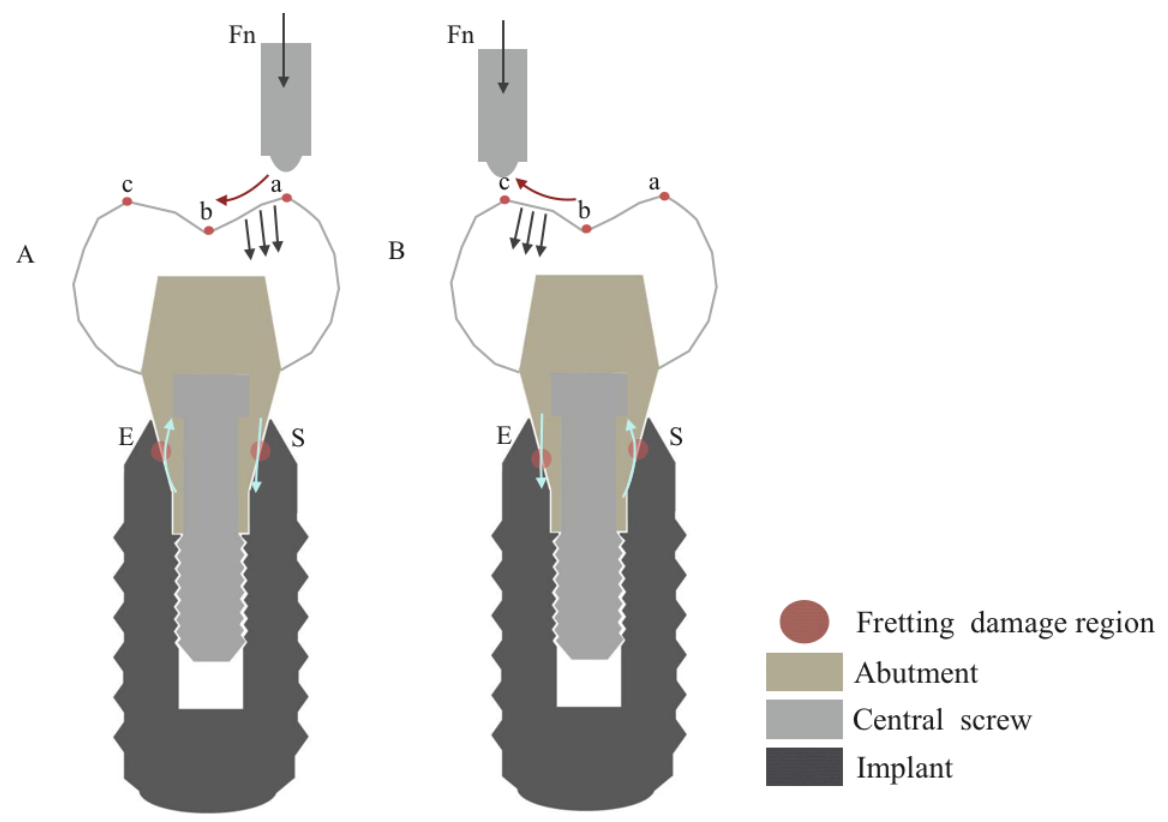

\begin{tabular}{ccccc}
\hline \multirow{2}{*}{ Groups } & \multicolumn{2}{l}{ FT } & & \multicolumn{2}{c}{ CW } \\
\cline { 2 - 5 } & $\mathrm{M} 1$ & $\mathrm{M} 2$ & $\mathrm{M} 1$ & $\mathrm{M} 2$ \\
\hline $\mathrm{D}_{\text {mix }}$ & 0.058 & 0.011 & 0.594 & 0.172 \\
$\mathrm{D}_{\max }$ & 4.158 & 1.323 & 10.826 & 12.048 \\
$\mathrm{D}_{50}$ & 0.433 & 0.128 & 2.915 & 3.143 \\
$\overline{\mathrm{D}}$ & 0.553 & 0.232 & 3.142 & 3.675 \\
\hline
\end{tabular}




\begin{tabular}{|c|c|c|c|c|}
\hline \multirow{2}{*}{ Groups } & \multicolumn{3}{|l|}{ FT } & $\mathrm{CW}$ \\
\hline & M1 & M2 & M1 & M2 \\
\hline$T_{n}$ & $31.88 \pm 0.21$ & $28.83 \pm 0.62$ & $31.57 \pm 0.30$ & $29.12 \pm 0.66$ \\
\hline $\mathrm{T}_{\mathrm{s}}-\mathrm{T}_{\mathrm{n}}$ & $3.12^{\mathrm{a}}$ & $6.17^{b}$ & $3.43^{\mathrm{a}}$ & $5.88^{\mathrm{b}}$ \\
\hline $\mathrm{T}_{\mathrm{m}}$ & $24.31 \pm 0.65$ & $21.15 \pm 0.76$ & $21.54 \pm 0.42$ & $20.68 \pm 0.47$ \\
\hline $\mathrm{T}_{\mathrm{s}}-\mathrm{T}_{\mathrm{m}}$ & $10.69^{+}$ & $13.85^{*}$ & $13.46^{++}$ & $14.32^{*}$ \\
\hline \multirow{2}{*}{ Groups } & FT & & & $\mathrm{CW}$ \\
\hline & M1 & M2 & M1 & M2 \\
\hline \multirow[b]{2}{*}{$\delta_{\mathrm{n}}$} & \multirow[b]{2}{*}{$8.91 \pm 0.6^{\mathrm{a}}$} & $17.63 \pm 1.77$ & \multirow[b]{2}{*}{$9.80 \pm 0.85^{\mathrm{a}}$} & $16.80 \pm 1.89$ \\
\hline & & b & & b \\
\hline \multirow{2}{*}{$\delta_{\mathrm{m}}$} & \multirow{2}{*}{$30.54 \pm 1.86^{+}$} & $39.57 \pm 2.17$ & $38.46 \pm 1.20$ & $40.91 \pm 1.34$ \\
\hline & & $*$ & + & $*$ \\
\hline
\end{tabular}

